\title{
Vacancy trapping and annealing in noble-metal films grown at low temperature
}

\author{
C. E. Botez, K. Li, E. D. Lu, W. C. Elliott, and P. F. Micelia) \\ Department of Physics and Astronomy, University of Missouri-Columbia, Columbia, Missouri 65211 \\ E. H. Conrad \\ School of Physics, Georgia Institute of Technology, Atlanta, Georgia 30332-0430 \\ P. W. Stephens \\ Department of Physics, State University of New York, Stony Brook, New York 11794
}

(Received 1 August 2002; accepted 16 October 2002)

\begin{abstract}
We have used synchrotron x-ray diffraction to study the homoepitaxial growth on $\mathrm{Cu}(001), \operatorname{Ag}(001)$, and $\operatorname{Ag}(111)$, at temperatures between 300 and $65 \mathrm{~K}$. The growth on all of these surfaces exhibits a consistent trend towards a large compressive strain that is attributed to the incorporation of vacancies into the growing film below $160 \mathrm{~K}$. In each case, the vacancy concentration is $\sim 2 \%$ at $110 \mathrm{~K}$ and we have measured the temperature dependence for incorporation on the (001) surfaces as well as the annealing behavior for $\mathrm{Cu}(001)$. These results, which suggest new kinetic mechanisms, have important implications for understanding epitaxial crystal growth. (c) 2002 American Institute of Physics. [DOI: 10.1063/1.1527988]
\end{abstract}

The design and fabrication of electronic nanostructures critically relies on the ability to understand and control the kinetic mechanisms that govern the epitaxial growth of thin films. Consequently, there is considerable interest ${ }^{1}$ to explore homoepitaxy where the absence of potential complications associated with strain or misfit dislocations (that would be present for heteroepitaxial systems) permit a fundamental investigation into the underlying mechanisms that dictate the surface morphology. Although theoretical studies and simulations have been quite successful ${ }^{2,3}$ in reproducing many of the features observed in experiments, the reliability of these theoretical predictions largely depends on knowing the specific kinetic mechanisms that are operative on the surface. It is, therefore, surprising that the role of vacancy formation in homoepitaxy has received little attention, in spite of recent simulations ${ }^{4,5}$ demonstrating kinetic channels that allow the formation of vacancies or voids in the growing film. These simulations proposed ${ }^{4}$ that "downward funneling" [a mechanism that has been employed to explain the reentrant smooth growth of (001) surfaces at low temperature] can be "restricted," causing the atomic mobility at step edges to be modified, thereby leading to overhang formation and vacancy incorporation. It was also suggested that vacancy formation strongly affects the surface morphology. Specifically, their formation was proposed to explain the reentrant rough growth observed at very low temperatures on $\operatorname{Ag}(001)$. Experimentally, vacancy formation and its connection to the surface morphology have not been established. Yet, it is obvious that if vacancies are incorporated into a growing film, the mechanisms leading to their formation are also ones that affect surface morphology. These mechanisms might also have important implications for growth at temperatures well above the vacancy formation temperature.

\footnotetext{
a) Author to whom correspondence should be addressed; electronic mail: micelip@missouri.edu
}

In this letter, we report the results of x-ray scattering experiments that demonstrate the striking similarity of vacancy incorporation during the low-temperature homoepitaxial growth on three noble-metal surfaces: $\mathrm{Ag}(111)$, $\mathrm{Ag}(001)$, and $\mathrm{Cu}(001)$. We also present the temperature dependence of vacancy incorporation for the (001) surfaces as well as the annealing behavior of the vacancies for $\mathrm{Cu}$. A detailed analysis of the different surfaces will be reported elsewhere. ${ }^{6,7}$ We suggest that vacancy incorporation is likely to be ubiquitous among many homoepitaxial growth systems, with important consequences for the surface morphology, i.e., the onset of vacancy incorporation occurs in the same temperature range where substantial changes in the growing surface have been observed for all three systems studied. ${ }^{3,6,8-10}$

The measurements on $\mathrm{Ag} / \mathrm{Ag}(001)$ were carried out on the Midwest Universities Collaborative Access Team ( $\mu \mathrm{CAT}$ ) beamline at the Advanced Photon Source, while Ag/ $\mathrm{Ag}(111)$ and $\mathrm{Cu} / \mathrm{Cu}(001)$ were measured using the SUNY X3B2 beamline at the National Synchrotron Light Source. In both cases the surface preparation, growth and x-ray scattering measurements were achieved with the sample contained in an ultrahigh vacuum (UHV) chamber (base pressure $10^{-10}$ Torr). The surfaces were prepared by several cycles of $\mathrm{Ar}^{+}$sputtering followed by high-temperature annealing. Prior to each deposition cycle, the quality of the starting surface was carefully assessed by x-ray scattering and Auger spectroscopy (AES). Within AES sensitivity no contaminants were present at any of the temperatures used in this study. The depositing atoms $(1 \mathrm{ML} / \mathrm{min})$ were evaporated from a thermal oven and the deposition was interrupted to allow $\mathrm{x}$-ray scattering measurements. The $\mathrm{x}$-ray reflectivity was obtained at different values of the surface-normal scattering wave vector, $Q_{z}$, by scanning across the specular rod and subtracting the diffuse component. ${ }^{11}$ 


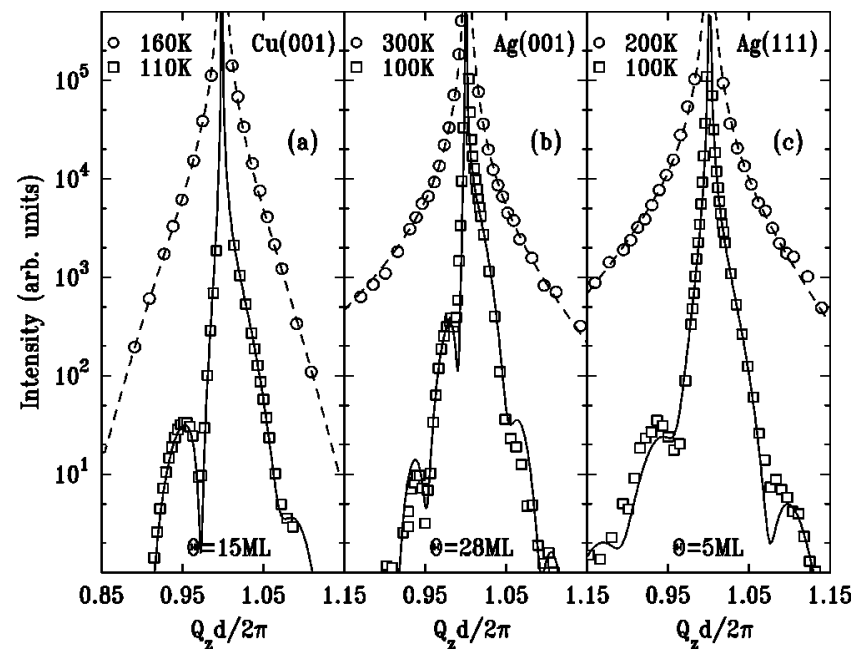

FIG. 1. Specular x-ray reflectivity from (a) $\mathrm{Cu} / \mathrm{Cu}(001)$, (b) $\mathrm{Ag} / \mathrm{Ag}(001)$, and (c) $\mathrm{Ag} / \mathrm{Ag}(111)$ films. At the higher temperatures, the data (open circles) are well described by a simple Gaussian surface roughness (dashed lines), whereas for $T \sim 100 \mathrm{~K}$ a real-space model that includes a large surfacenormal compressive strain in the deposited film is necessary to fit (solid lines) the interference fringes and the asymmetry about the Bragg reflection present in the measured reflectivity profiles (empty squares).

Figure 1 shows the effect of reducing the deposition temperature on the specular reflectivity from the homoepitaxially grown films. At higher temperatures, the measured reflectivity coincides extremely well with a real-space model where the structure of the film is identical to that of the bulk substrate and the surface is composed of a collection of terraces (steps) having a Gaussian distribution of heights. ${ }^{12}$ This type of analysis has been extensively used in our previous studies of metal homoepitaxy. ${ }^{10,12}$ At $100 \mathrm{~K}$, however, the reflectivity profiles measured around the Bragg reflection exhibit two new features: a pronounced asymmetry and interference fringes with a periodicity corresponding to the film thickness. Both features are well explained by a model ${ }^{6}$ where, in addition to some surface roughness, a surface-normal compressive strain:

$$
\frac{\Delta d}{d}=\frac{d_{\text {film }}-d_{\text {bulk }}}{d_{\text {bulk }}}
$$

is uniformly distributed through the deposited film. The solid lines in Fig. 1 are best fits to this model. The fits allow the strain to be determined from the reflectivity: at $100 \mathrm{~K}$, films grown on all three orientations typically exhibit $\Delta \mathrm{d} /(\mathrm{d}) \sim$ $-1 \%$.

We attribute the origin of this strain to the incorporation of vacancies or vacancy clusters because a substantial negative volume change is necessary to produce the observed large compressive strain. Other defects that might form at low temperature, such as stacking faults and dislocations, do not possess a sufficient negative volume change to account for the observed effect. It is also important to realize that impurity contamination due to low- $T$ adsorption can be excluded as the source of strain, since, a very large impurity concentration would be necessary to account for the magnitude of the observed strain, whereas AES spectra show clean surfaces before and after the deposition, at all temperatures. Moreover, the incorporation of larger substitutional impuriDownloaded 28 May 2010 to 128.206.162.204. Redistribution subje

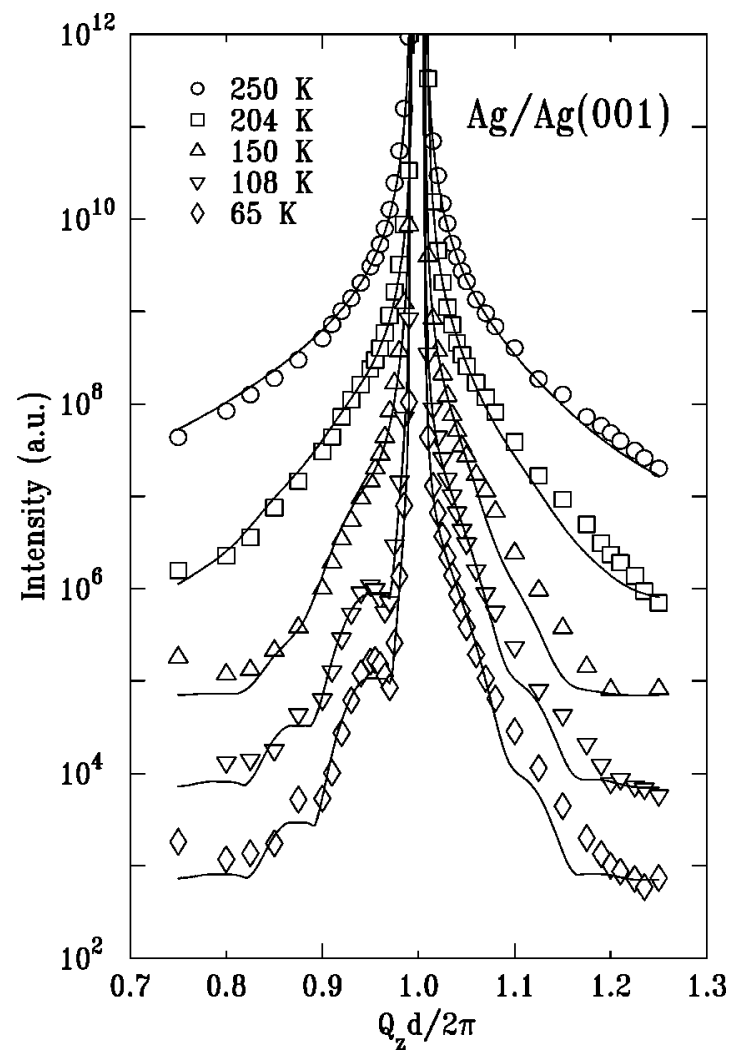

FIG. 2. Specular x-ray reflectivity measured for 15-ML-thick $\mathrm{Ag} / \mathrm{Ag}(001)$ films (empty symbols), deposited at different temperatures, between 65 and $250 \mathrm{~K}$. The solid lines are best fits to a model that includes a surface-normal strain produced by the incorporation of vacancies.

ties or small impurities that occupy interstitial sites would cause an expansion rather than the observed contraction of the lattice.

From the measured strain, we can estimate the vacancy concentration, $c_{v}$, assuming a point defect model: ${ }^{13}$

$$
\frac{\Delta d}{d}=-\alpha \cdot\left(1+2 \frac{C_{12}}{C_{11}}\right) \cdot c_{v} .
$$

Here, $\alpha=0.2$ relates the volume contraction to the vacancy concentration ${ }^{13}$ and $C_{12} / C_{11}$ is the ratio of elastic constants for the metal. ${ }^{14}$ From this relationship and the measured strain magnitude $(\sim 1 \%)$, we obtain $c_{v} \sim 2 \%$ for all three surfaces at $100 \mathrm{~K}$.

We now consider the temperature dependence of the vacancy concentration. Figure 2 shows the specular reflectivity measured around the (002) Bragg reflection for 15-ML-thick Ag films deposited on $\operatorname{Ag}(001)$ at different temperatures between 65 and $250 \mathrm{~K}$. We observe that the interference fringes and asymmetry about the in-phase position are very pronounced at 65 and $110 \mathrm{~K}$ but attenuate at $150 \mathrm{~K}$ and eventually vanish at $T>200 \mathrm{~K}$. This suggests that the strain magnitude and, equivalently, the vacancy concentration incorporated into the deposited films decreases with increasing $T$. Indeed, fits to the reflectivity data yield a vacancy concentration that follows this trend, as shown by the solid symbols in Fig. 3. Similar measurements and analysis were performed for 15-ML-thick $\mathrm{Cu}$ films grown on $\mathrm{Cu}(001)$ at temperatures ranging from 110 to $300 \mathrm{~K}$. The results for $\mathrm{Cu}$, also shown in Fig. 3, bear a striking resemblance to those observed for $\operatorname{Ag}(001)$. Finally, we find that for $\operatorname{Ag}(111)$, the to AIP license or copyright; see http://apl.aip.org/apl/copyright.jsp 


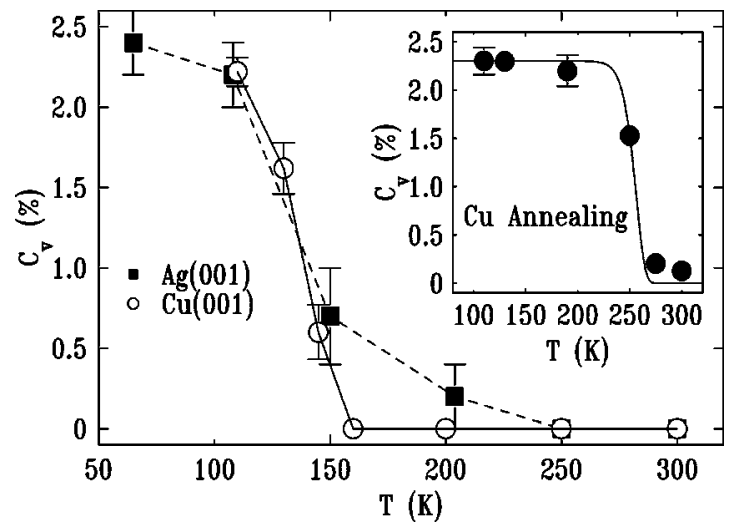

FIG. 3. Dependence of the vacancy concentration on the growth temperature, obtained from reflectivity measurements, is shown for $\mathrm{Ag} / \mathrm{Ag}(001)$ (filled squares) and $\mathrm{Cu} / \mathrm{Cu}(001)$ (empty circles) films. The inset shows the " $c_{v}$ vs $T$ " behavior for $\Theta=15 \mathrm{ML} \mathrm{Cu}$ films deposited on $\mathrm{Cu}(001)$ at $110 \mathrm{~K}$ and then annealed at progressively higher temperatures. As shown by the solid curve in the inset, given by, $c_{v}=c_{0} e^{-\alpha e^{-E_{b} / k_{B} T}}$, the known vacancy mobility for $\mathrm{Cu}$ agrees with the observed data. $E_{b}=0.72 \mathrm{eV}$ (Ref. 16) is the activation energy for monovacancy mobility in $\mathrm{Cu}$ and $\alpha=\pi^{2} t v / \Theta^{2}$, where $t \sim \frac{1}{2} \mathrm{~h}$ is the annealing time and $v=2 \times 10^{12} \mathrm{~Hz}$ is the attempt frequency that gives the best fit.

vacancies anneal out upon warming to room temperature. The inset of Fig. 3 shows the vacancy concentration for 15ML-thick $\mathrm{Cu} / \mathrm{Cu}(001)$ films grown at $110 \mathrm{~K}$ that were subsequently annealed at a higher temperature. These results are essentially identical to the well-known annealing behavior of vacancies observed in radiation damage studies of bulk copper: ${ }^{15,16} c_{v}$ exhibits a plateau between 100 and $200 \mathrm{~K}$ followed by a precipitous decrease between 200 and $300 \mathrm{~K}$. The curve in the inset was calculated using a $0.72 \mathrm{eV}$ activation energy that is known ${ }^{16}$ for monovacancy mobility in $\mathrm{Cu}$. This is further proof of the inclusion of vacancies in thin metal films grown at low temperatures.

The similarity of the growth behavior for the (001) orientations of $\mathrm{Ag}$ and $\mathrm{Cu}$ is evident not only in the incorporation of vacancies, but also extends to the reentrant smooth growth that has been observed ${ }^{3,8-10}$ in both of these systems below $200 \mathrm{~K}$. This suggests similar, if not identical, surface kinetics for these two systems. Finally, we point out that the mechanisms for vacancy formation should be quite general and not limited to homoepitaxy on (001) crystal faces, as shown by our finding of a $2 \%$ vacancy concentration incorporated during $\mathrm{Ag} / \mathrm{Ag}(111)$ growth at $100 \mathrm{~K}$. Moreover, we have observed ${ }^{6}$ substantial changes in the growing $\mathrm{Ag}(111)$ surface morphology whereby a bimodal distribution of surface heights emerges concomitantly with the formation of vacancies. Thus, the three noble metal systems studied here present strong evidence that the formation of vacancies is intimately tied to changes in the evolving surface morphology.
In summary we have used $\mathrm{x}$-ray reflectivity to study the temperature-dependent homoepitaxial growth on noble-metal surfaces. For three different systems, $\mathrm{Cu} / \mathrm{Cu}(001), \mathrm{Ag} /$ $\mathrm{Ag}(001)$, and $\mathrm{Ag} / \mathrm{Ag}(111)$, we observed that growth at low temperatures leads to the incorporation of an appreciable vacancy concentration, $c_{v}$, in the deposited film. $c_{v}$ is coverage independent, but strongly depends on the growth temperature, decreasing with increasing $T$ from $\sim 2 \%$ to zero within a narrow temperature interval.

Support is acknowledged from the NSF under Contract Nos. (P.W.S) DMR-9202528 and (P.F.M), (C.E.B) DMR9623827, MISCON under DOE Grant No. DE-FG0290ER45427, and the University of Missouri Research Board. The SUNY X3 beam line is supported by the DOE, under Contract No. DE-FG02-86ER45231 and the NSLS is supported by the DOE, Division of Material Sciences and Division of Chemical Sciences. The Advanced Photon Source is supported by DOE Contract No. W-31-109-Eng-38, and the $\mu$ CAT beam line is supported through the Ames Laboratory Contract No. DOE W-7405-Eng-82.

${ }^{1}$ See for example, Morphological Organization in Epitaxial Growth and Removal, edited by Z. Zhang and M. G. Lagally (World Scientific, Singapore 1998).

${ }^{2}$ J. G. Amar, Phys. Rev. B 60, R11317 (1999); M. Siegert, Phys. Rev. B 81, 5481 (1998); L. H. Tang, P. Smilauer, and D. D. Vvendensky, Eur. Phys. J. B 2, 409 (1998)

${ }^{3}$ C. R. Stoldt, K. J. Caspersen, M. C. Bartelt, C. Jenks, J. W. Evans, and P. A. Thiel, Phys. Rev. Lett. 85, 800 (2000).

${ }^{4}$ K. J. Caspersen and J. W. Evans, Phys. Rev. B 64, 075401 (2001).

${ }^{5}$ F. Montalenti and A. F. Voter, Phys. Rev. B 64, 081401 (2001).

${ }^{6}$ C. E. Botez, W. C. Elliott, P. F. Miceli, and P. W. Stephens, Phys. Rev. B 66, 075418 (2002).

${ }^{7}$ C. E. Botez, P. F. Miceli, and P. W. Stephens, Phys. Rev. B (accepted for publication)

${ }^{8}$ G. Constantini, F. Buatier de Mongeot, C. Boragno, and U. Valbusa, Surf. Sci. 459, L487 (2000).

${ }^{9}$ H.-J. Ernst, F. Fabre, R. Folkerts, and J. Lapujoulade, Phys. Rev. Lett. 72, 112 (1994).

${ }^{10}$ C. E. Botez, W. C. Elliott, P. F. Miceli, and P. W. Stephens, Phys. Rev. B 63, 113404 (2001).

${ }^{11}$ I. K. Robinson and D. J. Tweet, Rep. Prog. Phys. 55, 559 (1992).

${ }^{12}$ W. C. Elliott, P. F. Miceli, T. Tse, and P. W. Stephens, Physica B 221, 65 (1996); Phys. Rev. B 54, 17938 (1996).

${ }^{13}$ C. W. Tucker and J. B. Sampson, Adv. Cryog. Eng. 2, 433 (1954); T. Broom and R. K. Ham in Institute of Metals Monograph and Report Series No. 23 (Institute of Metals, London, 1958), pp. 41.

${ }^{14}$ The second term containing the elastic constant ratio, $C_{12} / C_{11}=0.76$ for $\mathrm{Ag}$ and 0.72 for $\mathrm{Cu}$, accounts [P. F. Miceli et al., Appl. Phys. Lett. 58, 1602 (1991)] for the lateral clamping of the film that is reported in Ref. 6.

${ }^{15}$ P. Ehrhart, K. H. Robrock, and H. R. Schober, in Physics of Radiation Effects in Crystals, edited by R. A. Johnson and A. N. Orlov (Elsevier Science, Amsterdam, 1986), pp. 60.

${ }^{16}$ R. W. Balluffi, J. Nucl. Mater. 69\&70, 240 (1978). 